\title{
El aprendizaje de los estudiantes de primero de carrera evaluado en ocho indicadores
}

\author{
*Carmen Delia García-Fuentes de la Fuente. **Nuria Manzano Soto \\ *Universidade da Coruña, **Universidad Nacional de Educación a Distancia
}

\begin{abstract}
Resumen
En este artículo, se realiza una presentación del tema del aprendizaje en alumnos de primero de carrera universitaria. El tema del aprendizaje se introduce desde la concepción clásica de enfoques de aprendizaje, se menciona el conectivismo de Siemens (2006) por ser un enfoque más adaptado al aprendizaje de la era digital en la que nos encontramos inmersos. Finalmente comentamos los resultados obtenidas en el cuestionario adaptado del de Monereo (1993) sobre autoconocimiento en la forma de estudio. Se comentan los resultados obtenidos en la evaluación de necesidades de los estudiantes de la muestra.
\end{abstract}

Palabras clave: Aprendizaje, autoconocimiento y estrategias de estudio.

\section{Introducción}

Existe un interés creciente, manifestado por los propios estudiantes, en comprobar cual es su forma de aprendizaje y cuanto suponen las estrategias de estudio que conocen y practican, en el aumento de conocimientos y en el éxito de su rendimiento académico. No pocos se acercan a los Centros de Orientación y Empleo (COIEs) para contrastar con el experto, su forma personal de aproximarse al aprendizaje.

En general, los estudiantes, en sus actividades académicas y vitales, buscan aprender. A esto llegan con la idea de que estudiar, al igual que asistir a clase o trabajar intelectualmente les va a conducir a la consecución del aprendizaje deseado. Casi siempre se identifica el aprendizaje como conjunto de habilidades exigidas para hacer algo.

Es preciso destacar que la mayoría de los estudiantes persiguen con el estudio objetivos similares: intentan adquirir información, intentan adquirir destrezas a nivel individual y en su funcionamiento cooperativo e intentan utilizar el autoconocimiento de las capacidades propias con objeto de poder utilizarlas lo mejor posible.
En este sentido Bernard (1995) identifica la adquisición de la información respecto a fenómenos, hechos, conceptos, principios, con los contenidos de las asignaturas objeto de estudio. Así mismo equipara el propósito de adquirir destrezas para la aproximación a los contenidos de estudio con las llamadas estrategias de estudio-aprendizaje. Finalmente, el interés por el autoconocimiento de las estrategias que utiliza, estriba en intentar identificar sus propias capacidades, conocimiento de sí mismo y la reflexión sobre su propia forma de aprendizaje es decir el conocimiento llamado metacognitivo.

En los estudiantes en general y en los que acuden por primera vez a la Universidad también, es preciso diferenciar si su proceso de aprendizaje puede ser identificado con un aprendizaje eficaz. A su vez existe otro factor a considerar relacionado con la eficacia y el aprendizaje a este suele identificársele como rendimiento académico.

En este artículo, interesa reflexionar sobre cual es el tipo de proceso de aprendizaje que recorre el estudiante y también desde qué enfoque aborda su devenir en la universidad.

Si se parte de la consideración clásica de Biggs (1988) y Entwistle (1988) sobre las aproximaciones al aprendizaje de los estudiantes y aprendices, nos encontramos con tres enfoques en principio: el aprendizaje superficial, el aprendizaje profundo y el aprendizaje estratégico.

Bernard (1995), Recio M.A. y Cabero J. (2005), caracterizan el enfoque superficial como una forma de aprender sin relacionar unos conocimientos con otros, realizan memorización aislada, los estudiantes no se preocupan de distinguir lo importante de lo secundario, utilizan las fuentes de información recomendadas explícitamente, de esta forma, el único objetivo que tienen suele ser aprobar.

Respecto al aprendizaje profundo, Bernard (1995) lo identifica como la forma de relacionar la tarea con otros conocimientos, interpretar personalmente lo que se estudia, utilizar diferentes fuentes de información y 
estructurar lo que se aprende, entre otras muchas funciones cognitivas.

El enfoque estratégico o de logro, lo caracterizan, González-Pienda, Roces, Bernardo y García (2002) como la forma de plantearse el aprendizaje de aquellos estudiantes que necesitan un alto rendimiento para incrementar la autoestima, sobresalir y competir.

Es adecuado indicar que los tres enfoques de aprendizaje enunciados, pueden considerarse clásicos, dentro de su alto nivel de importancia. Suele coincidir que la mayoría de los estudiantes se manejan con los tres enfoques de manera alternativa o simultánea, según la conveniencia y adecuación a la situación presentada. Otros grupos de estudiantes presentan prevalencia de un tipo de aprendizaje sobre el otro. Otras corrientes que se incluyen en la práctica del aprendizaje en general y en el aprendizaje de estudio en particular, son las relativas a la forma de relacionar unos conocimientos con otros.

Dado que el conductismo, el cognitivismo y el constructivismo, son grandes teorías de aprendizaje utilizadas sobre todo en ambientes instruccionales, entre muchas otras concepciones, también es preciso mencionar, por la actualidad que representa y la oportunidad, el llamado Conectivismo, teoría de aprendizaje más adaptada a la era digital. Su creador, George Siemens (2006) Universidad de Manitoba (Canadá), advierte de esta nueva forma de aprender, centrada en las posibilidades de la sociedad actual. Señala que aprender en la sociedad de la información debe realizarse de forma diferente. Esta idea es el eje de su publicación titulada conociendo el conocimiento, Tal como señala Siemens (2006) para aprender es necesario establecer conexiones entre fuentes y redes de información, que cambian y crecen sin cesar.

Conviene consultar el funcionamiento de los sistemas de información de adolescentes, ya estos están más próximos a un proceso de formación y el de las personas adultas cuando intentan poner en práctica estrategias de aprendizaje. En este sentido, algunas investigaciones entre ellas la referida por Salmerón, Cerdan \& Naumam (2015) señalan que en la búsqueda de información a través de redes informáticas, para responder a preguntas, los adolescentes mayores, seleccionaban mejor los canales de información cuando se les proporcionaban conocimientos previos sobre la temática preguntada, de esa forma no se distraían seleccionando y siguiendo canales irrelevantes. Conviene destacar el rol de orientación en el aprendizaje que requiere esta tarea.

En una primera impresión parece suceder que los adolescentes y los estudiantes adultos, están incorporando en gran medida canales de información diversos y diversificados a sus estrategias de aprendizaje.

\section{Autoconocimiento y estrategias de estudio}

Desde la enseñanza y los estudios actuales, en el abanico instruccional en la Universidad Española, es fácil distinguir varias dimensiones, todas ellas relacionadas con el almacenamiento de la información, la codificación y organización de la información y la metacognición. En todos estos planteamientos de aprendizaje y estudio ocupa un lugar preponderante la eficacia, es decir el aprendizaje eficaz.

Así pues, un estudiante eficaz, reflexiona sobre su capacidad intelectual y competencia intelectual, sobre su capacidad para la planificación, sobre su atención, sobre su motivación para estudiar, sobre su autoestima y autoconfianza, sobre su posibilidad de esforzarse, si fuera preciso, sobre su posibilidad de rendir adecuadamente en los exámenes.

Interesa señalar la conveniencia de utilizar para un estudio eficaz, técnicas y estrategias suficientemente coordinadas para el éxito en el aprendizaje y el estudio. Algunos autores, entre ellos Bernard (1995), identifica las estrategias como planes de actividad compleja en la que se identifican conjuntos de acciones realizadas sucesiva y ordenadamente con vistas a la consecución de una meta. En cambio las técnicas son consideradas como cada una de las pequeñas metas y acciones que hay que realizar para llevar a cabo una estrategia. Unos aspectos y otros interaccionando debidamente, colaboran para alcanzar la posibilidad de ser un estudiante estratégico y altamente productivo, o mejor, altamente eficaz.

\section{Aplicación práctica}

Con objeto de proporcionar a cada estudiante unas pautas para su autoconocimiento en relación a su respuesta ante el estudio. Nos planteamos realizar un estudio piloto sobre una muestra no representativa de los estudiantes de primero de carrera universitaria y Curso de Acceso, de la Universidad Nacional de Educación a Distancia (UNED) Centro Asociado de A Coruña.

El objetivo planteado consiste en evaluar las necesidades de formación en estrategias de estudio.

El número de sujetos de la muestra es de 123. Muchos de los sujetos de la muestra tienen jornada laboral completa y además realizan estudios universitarios. Se trata por tanto de una muestra incidental en la que la variable sexo, refiere que el $63 \%$ representado son mujeres frente al $37 \%$ de varones. 
Se realiza la evaluación de necesidades a través del Cuestionario de Monereo (1995), Aprendo a conocerme como estudiante, se han adaptado algunos ítems, a una población de universitarios mayores y a una enseñanza en la que se utilizan plataformas de aprendizaje. Dicho cuestionario consta de ocho bloques, cada bloque tiene ocho ítems y cada item cuenta con 5 alternativas cualitativas de respuesta. Estas cinco alternativas se corresponden cuantitativamente con valores entre 1 , nunca o casi nunca, 2, pocas veces, 3 , algunas veces, 4 , frecuentemente y 5 siempre. Todos los apartados del Cuestionario aplicado avanzan del item $n^{\circ} 1$ al item $n^{\circ} 8$ de una forma progresiva.

El propio cuestionario, Aprendo a conocerme como estudiante, de Monereo (1994), da las claves para la autoevaluación de cada apartado. Así indica a los estudiantes que a medida que se acerquen a los 40 puntos en cada apartado pueden considerarse buenos estudiantes. También indica que las puntuaciones totales por apartado están comprendidas entre los 20-25 puntos.

Los bloques se enumeran a continuación con la explicación sintética del enunciado global con el que han sido identificados para el tratamiento de datos con el paquete estadístico SPSS.

A. Interés general por aprender

B. Organización y planificación del estudio.

C. Atención y esfuerzo.

D. Memorización.

E. Lectura comprensiva de estudio.

F. Estudio personal en casa.

G. Exámenes.

H. Autoconfianza y autoestima.

A continuación se constatan los valores obtenidos en cada uno de los ocho apartados que resultan de aplicar dicho instrumento a la muestra de estudiantes testados.

También hacemos constar, en algunos de los bloques, las desviaciones típicas de los ítems cuyos resultados, parece relevante comentar.

\section{Estudios}

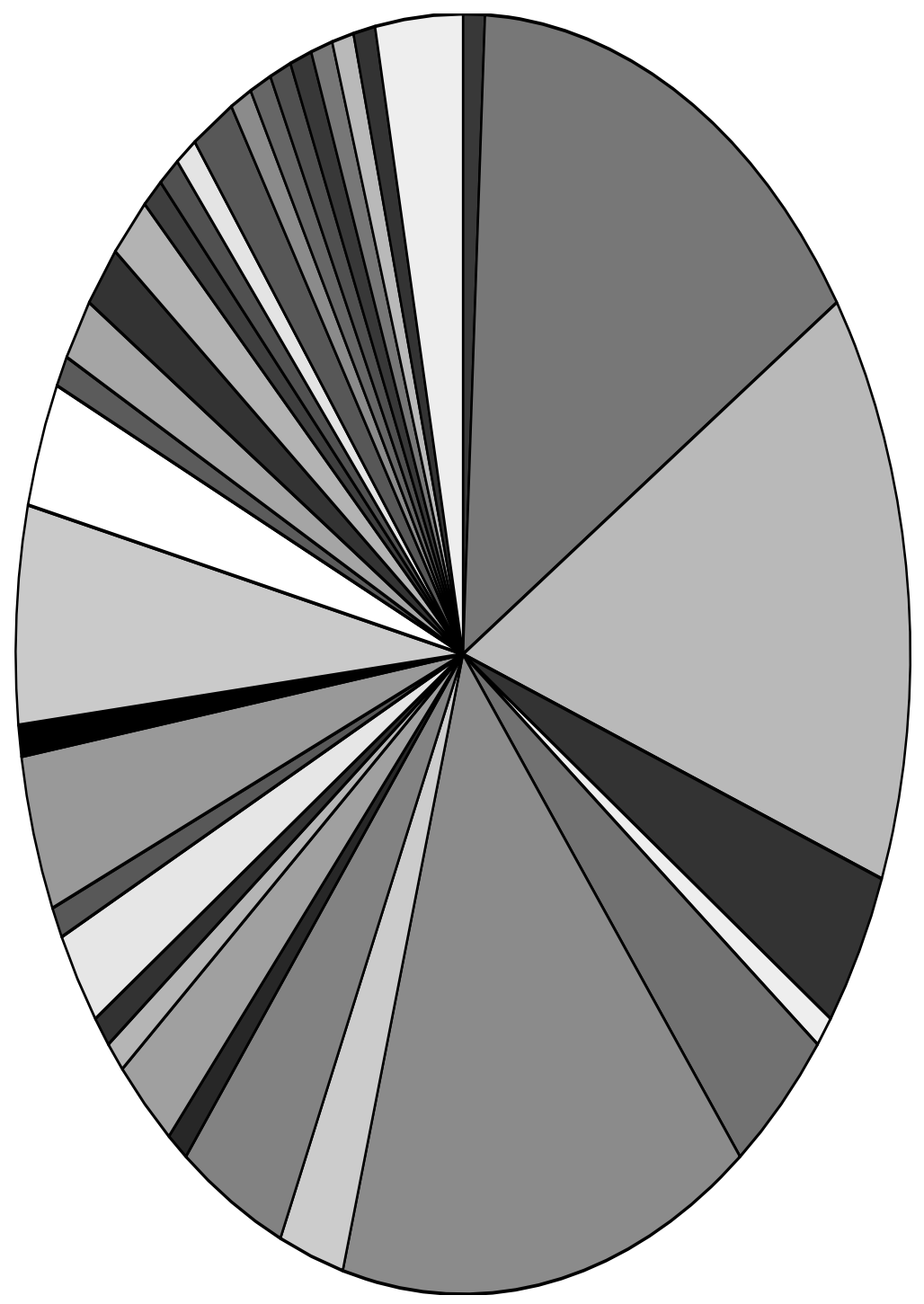

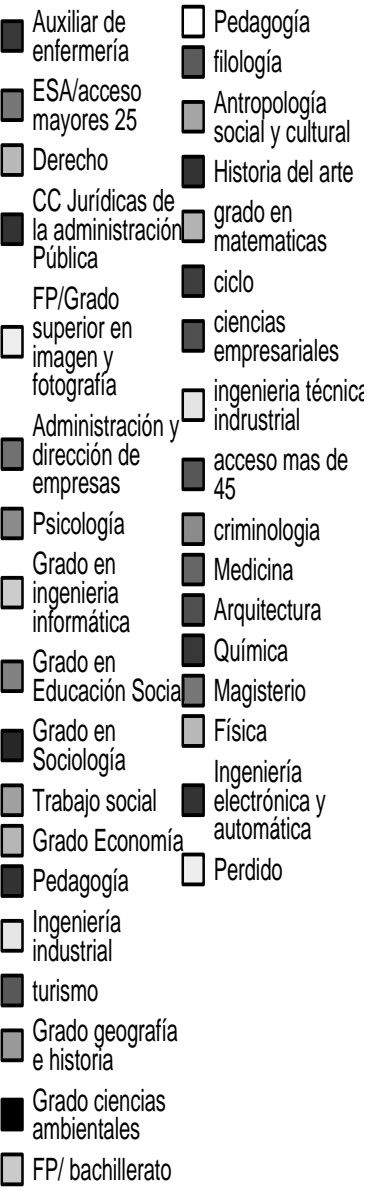


A la vista de la muestra, la variedad de procedencia, filiación de estudios y carreras de la misma, constatamos que revisando la tabla de frecuencias que da lugar al gráfico precedente, se observa que la mayor frecuencia está en el Acceso de Mayores de 25 y las Carreras de Psicología, Derecho, este $\mathrm{n}^{\circ}$ de frecuencias en el cómputo total, representa un $18 \%$ para Acceso mayores de 25, un 18\%, Psicología un 18\% y Derecho un $18 \%$, que conforman un $50 \%$ del total.

Analizando ahora los apartados del Cuestionario en las respuestas dadas a los ítems y la media, obtenida con esas puntuaciones, nos encontramos lo que indicamos a continuación:

En el apartado A, de interés por aprender, la media más alta le corresponde al item $n^{0} 5$ cuyo contenido versa sobre el beneficio que reporta el estudio, al que dan un valor de 4.63 y una desviación típica de .063.En este mismo bloque, la media más baja de 3.47 , le corresponde al item $n^{\circ} 3$ de entusiasmo e interés por el estudio, en este caso la desviación típica es de 1.007.

En el apartado B, la media más elevada, le corresponde al item $\mathrm{n}^{\circ} 6$ de asignar prioridades para la acción y es de 3.72 y una desviación típica de 1.119, en cambio la más baja de 2.8, le corresponde al item cuyo contenido es evaluarse a sí mismo y este presenta una desviación típica de 1.140.

En el apartado C, el item 1 atención en clase y el 2 de preguntar lo que no entienden, arrojan idéntica media, 3.95, comentamos sólo el 1, ya que tiene una desviación típica de 0.825. La media más baja estuvo en el de estudiar y participar de forma activa, con $2.80 \mathrm{y}$ desviación típica de 1,119.

El apartado D, memoria, la media más alta recayó en el el $n^{\circ} 1$ de entender bien lo que lee y escucha, con 3.93 y una desviación típica de 0.669. La menor puntuación, media $1.80 \mathrm{y}$ desviación típica de 1.133 y corresponde a la baja utilización de procedimientos electrónicos para implementar técnicas de memorización.

El apartado E, la media más elevada, resultó el $\mathrm{n}^{\circ} 5$, cuyo contenido es relacionar lo leído con lo que ya sabe, y alcanza un 4.06 con una desv típica de 0.902 .

El apartado F, el más elevado valor resultó en el item $n^{\circ} 7$ relacionado en el orden en los materiales de trabajo y estudio, con 4.30 y una desviación de 0.48 .

El apartado $G$, el resultado más elevado fue obtenido por el item el $\mathrm{n}^{\circ} 4$, una media de 3.29 y una desviación de 1.128, su contenido es adelantar e intentar adivinar preguntas en los exámenes, En cambio la media más baja estuvo en el item $n^{\circ} 7$ sobre aplicar estrategias de respuesta en los exámenes su valor es de 2.76 con una desviación de 1.162.

Finalmente en el apartado $\mathrm{H}$, el item $\mathrm{n}^{\circ} 7$, sobre capacidad de decisión, es en el que han alcanzado mejor media con 3.79. y una desviación de 1.009. En cambio la media más baja se muestra en dos ítems, saber superar los fallos en el rendimiento, item $\mathrm{n}^{\circ} 4$ y sentirse seguro cuando se habla ante los demás, item $\mathrm{n}^{\circ}$ 8 .La media alcanza 3.20, referenciamos, el numero 4 ya que su desviación típica es la menor de las dos encontradas.

En resumen, la media más alta obtenida comparando los ocho apartados del Instrumento de evaluación adaptado de Monereo (1993) resulta que se sitúa en el apartado F, titulado estudio personal y hace relación al orden en los materiales de trabajo y a los apuntes. La máxima carencia la encontramos en el apartado de planificación respecto a la cultura de autoevaluación y registro diario.

La dificultad para reflexionar de forma metódica sobre las dificultades de la tarea que nos ocupa, y la toma de decisiones sobre el dilema de cuantas de esas dificultades pueden resolver por si mismos, confiere un punto de inflexión en cualquier estudiante que se enfrenta cada día a un nuevo reto. Esto sucede frecuentemente en estudiantes de la UNED. Ellos compaginan el estudio y otras ocupaciones laborales y familiares y a menudo empiezan o retoman estudios en un tiempo distante a l que los habían iniciado.

Respecto al item en el que destacamos la máxima carencia en esta evaluación de necesidades de estrategias de trabajo intelectual, mencionado anteriormente, habría que concluir que cuestiones como esta, quizá tengan unas implicaciones mucho más profundas que deberían ser trabajadas por cada estudiante a nivel personal. Sería posible afrontarlas dentro un proceso de crecimiento personal, apoyados en el COIE más próximo y los programas de mentoring que están en implantación, sin olvidar otras muchas acciones de asesoramiento disponibles.

\section{A modo de síntesis}

A lo largo de este artículo, se ha realizado una presentación del tema del aprendizaje en alumnos de primero de carrera universitaria. Se presentó el tema del aprendizaje en la introducción y se mencionó el conectivismo de Siemens (2006) por ser un enfoque más adaptado al aprendizaje de la era digital en la que nos encontramos inmersos. Finalmente comentamos los resultados obtenidas en el Cuestionario adaptado de Monereo (1993) aplicado a alumnos de primero de carrera universitaria. 


\section{Referencias bibliográficas}

Ausubel, D.P.;Novvak,J y Hanesian,H. (1978): Educational Psychology: A cognitiveview.New York: Holt, Rineart. (Traducción cast. Psicología Educativa un punto de vista cognoscitivo. México: Trillas, 1983)

Bernard Mainard, J.A. Estrategias de estudio en la universidad. Madrid. Síntesis S.A.

Biggs, J.B.( 1988): Assessing student approaches to learning .australian Psychologist, 23, 197-206.

Bruner(1988): Desarrollo cognitivo y educación. Madrid. Morata.

Entwistle, N.J.(1988): La concepción del aprendizaje en el aula. Barcelona.Paidós

González-Pienda, Roces. Bernardo y García (2002). Estilos de aprendizaje y estilos de pensamiento. En J.A. González Pienda, R González Cabanach, J.C. Nuñez y A . Valle Arias Eds. Manual de Psicología de la Educación (pp. 165-180). Madrid. Pirámide.

Monereo Font, C. y Castelló Badía, M.(1997): Las estrategias de aprendizaje. Barcelona Ed Edbé.
Monereo, C. (Comp.) (1993). Las Estrategias de Aprendizaje: procesos, contenidos e interacción. Barcelona: Doménech Edicions.

Recio M.A. y Cabero J. (2005): Enfoques de aprendizaje, rendimiento académico y satisfacción de los alumnos en la formación en entornos virtuales. Pixel-Bit $\mathrm{n}^{\circ} 25$ enero. http://www.sav.us.es/pixelbit/pixelbit/articulo s/n25/n25art/art2510.htm

Salmerón, L., Cerdan, R.\& Naumam, J. (2015): Como navegan los adolescentes en Wikipedia para contestar a preguntas. Infancia y aprendizaje 2015;

Skinner B.F.( 1973 ): Psicología de la Educación .Madrid. Ed Morata

Siemens, G. (2006): Conociendo el conocimiento. Traducida 2010 Quintana,

Vidal, Torres y Castrillejo. Ilustrada por Santamaría y Alonso. Licencia Creative Commons disponible en http://www.nodosele.com/editorial, consultada 29/06/2015. 